\title{
KERNEL-TYPE ESTIMATION OF BIVARIATE DISTRIBUTION FUNCTION FOR ASSOCIATED RANDOM VARIABLES
}

\author{
C. Azevedo ${ }^{1}$ and P. E. Oliveira ${ }^{2}$ \\ ${ }^{1}$ Universidade do Minho, Braga, Campus de Gualtar, 4700 Braga, Portugal \\ e-mail: cecilia@math.uminho.pt \\ ${ }^{2}$ Universidade de Coimbra, Portugal \\ e-mail: paulo@mat.uc.pt
}

April 29, 2005

\begin{abstract}
Let $X_{n}, n \in \mathbb{N}$, be a stationary sequence of associated random variables with uniform distribution on $[0,1]$ and $F$ the distribution function of $\left(X_{1}, X_{k+1}\right)$, for fixed $k \in \mathbb{N}$. We introduce a kernel estimator for $F$ and study its asymptotic properties and moments, characterizing their convergence rates. From these we derive the optimal rate for the bandwidth, which is of order $n^{-1}$. Conditions are also given to ensure that the finite dimensional distributions are asymptotically gaussian.
\end{abstract}

Keywords: association; stationarity; kernel estimation; convergence

\section{INTRODUCTION}

In this article we study the estimation of a bidimensional distribuition function based on associated samples. This problem was motivated by the need to characterize the covariance function of the limiting process of the sequence of empirical processes

$$
Z_{n}(t)=\frac{1}{\sqrt{n}} \sum_{i=1}^{n}\left(\mathbb{I}_{[0, t]}\left(X_{i}\right)-t\right), \quad t \in[0,1] .
$$

where $X_{n}, n \in \mathbb{N}$, are uniformly distributed on $[0,1]$. This assumption on the distribution of the $X_{n}, n \in \mathbb{N}$, means no loss of generality, as the general case is derived from this one using the corresponding quantile function. plays 
For independent underlying variables $X_{n}, n \in \mathbb{N}$, it is well known that $Z_{n}$ converges in distribution to a brownian bridge in the Skhorohod space $D[0,1]$. Extensions to mixing or associated underlying variables of this convergence exist in the literature. In each of these cases, $Z_{n}$ converges in distribution to a gaussian centered process with covariance function

$$
\begin{aligned}
& \Gamma(x, y)=\min (x, y)-x y+ \\
& \quad+\sum_{k=2}^{\infty}\left[\left(\mathrm{P}\left(X_{1} \leq x, X_{k} \leq y\right)-x y\right)-\left(\mathrm{P}\left(X_{1} \leq y, X_{k} \leq x\right)-x y\right)\right] .
\end{aligned}
$$

A first approach on approximating the series consists on estimating each term $\mathrm{P}\left(X_{1} \leq x, X_{k} \leq y\right)$, thus suggesting the problem under study. The same problem has been addressed in Roussas (1995) and Cai, Roussas (1998) for unidimensional distribution functions based on associated samples. For higher dimensional spaces, the problem has been considered by Jin, Shao (1999) based on independent samples. For the same framework as in this article see Henriques, Oliveira (1999) for the properties of the histogram estimator.

Let us now define association. The variables $X_{n}, n \in \mathbb{N}$, are associated if, given $n \in \mathbb{N}$ and $f, g: \mathbb{R}^{n} \longrightarrow \mathbb{R}$ coordinatwise increasing,

$$
\operatorname{Cov}\left(f\left(X_{1}, \ldots, X_{n}\right), g\left(X_{1}, \ldots, x_{n}\right)\right) \geq 0
$$

whenever this covariance exists. For such variables, the covariances $\operatorname{Cov}\left(X_{i}, X_{j}\right)$ completely determine the dependence structure (see Newman (1984)). Thus it is natural to impose conditions on the decrease rate of such covariances.

\section{DEFINITIONS AND ASSUMPTIONS}

As described above, we are interested on the estimation of $F(x, y)=\mathrm{P}\left(X_{1} \leq\right.$ $\left.x, X_{k+1} \leq y\right)$ with $k$ fixed. A natural estimator would be

$$
\widehat{\varphi}_{n}(x, y)=\frac{1}{n-k} \sum_{i=1}^{n-k} \mathbb{1}_{[0, x] \times[0, y]}\left(X_{i}, X_{k+i}\right),
$$

studied in Henriques, Oliveira (1999). Here we will study the kernel estimator of $F$ defined by

$$
\begin{aligned}
\widehat{F}_{n}(x, y) & =\frac{1}{n-k} \sum_{i=1}^{n-k} U\left(\frac{x-X_{i}}{h_{n}}, \frac{y-X_{k+i}}{h_{n}}\right)= \\
& =\int_{\mathbb{R}^{2}} U\left(\frac{x-s}{h_{n}}, \frac{y-t}{h_{n}}\right) \widehat{\varphi}_{n}(d s, d t)
\end{aligned}
$$

where $U$ is a given distribution function and $h_{n} \longrightarrow 0$.

We now introduce the set of assumptions that will be referenced to throughout the text. 
(A1) $X_{n}, n \in \mathbb{N}$, is a strictly stationary sequence of associated variables with uniform distribution on $[0,1]$;

(A2) $k$ is a fixed integer and $F$ the distribution function of $\left(X_{1}, X_{k+1}\right) . F$ has bounded and continuous derivatives of first and second orders;

(A3) let $F_{j}$ be the distribution function of $\left(X_{1}, X_{k+1}, X_{j}, X_{k+j}\right) . F_{j}$ has bounded and continuous derivatives of first and second orders;

(A4) $U$ is twice differentiable. If $u=\frac{\partial^{2} U}{\partial x \partial y}$, it satisfies

$$
\begin{aligned}
& \int_{\mathbb{R}^{2}} x u(x, y) d x d y=\int_{\mathbb{R}^{2}} y u(x, y) d x d y=0 \\
& \int_{\mathbb{R}^{2}} x^{2} u(x, y) d x d y, \int_{\mathbb{R}^{2}} y^{2} u(x, y) d x d y<\infty ;
\end{aligned}
$$

(A5) the sequence of bandwidths is such that $n h_{n}^{2} \longrightarrow 0$;

(A6) $\sum_{j=1}^{\infty} j \operatorname{Cov}^{1 / 3}\left(X_{1}, X_{j}\right)<\infty$;

(A7) $V=\frac{\partial^{2} U^{2}}{\partial x \partial y}$ satisfies $\int_{\mathbb{R}^{2}} x^{2} V(x, y) d x d y, \int_{\mathbb{R}^{2}} y^{2} V(x, y) d x d y<\infty$.

Conditions (A1), (A2), (A4) and (A6) have already been used in Cai, Roussas (1998). Note further that (A6) implies

(A6') $\sum_{j=1}^{\infty} \operatorname{Cov}^{1 / 3}\left(X_{1}, X_{j}\right)<\infty$

which implies the convergence of $Z_{n}$ on $L^{2}[0,1]$ (see Oliveira, Suquet (1995)).

\section{CONSISTENCY OF THE ESTIMATOR}

The consistency of $\widehat{F}_{n}$ is established in two steps: $\widehat{F}_{n}$ verifies a strong law of large numbers and is asymptotically unbiased. To achieve this goal we need to study the behaviour of the covariance between each term in the sum (1), establishing their limits and convergence rates. Before looking at these covariances we shall characterize $\mathrm{E}\left[\widehat{F}_{n}(x, y)\right]$.

Theorem 3.1 Suppose the variables $X_{n}, n \in \mathbb{N}$, satisfy (A1), (A2) and (A4). Then, for each $x, y \in[0,1]$,

$$
\begin{aligned}
& \mathrm{E}\left[\widehat{F}_{n}(x, y)\right]=F(x, y)+\frac{h_{n}^{2}}{2}\left[\frac{\partial^{2} F}{\partial x^{2}}(x, y) \int s^{2} u(s, t) d s d t+\right. \\
& \left.\quad+\frac{\partial^{2} F}{\partial x \partial y}(x, y) \int s t u(s, t) d s d t+\frac{\partial^{2} F}{\partial y^{2}}(x, y) \int t^{2} u(s, t) d s d t\right]+o\left(h_{n}^{2}\right)
\end{aligned}
$$


Proof : As $\mathrm{E}\left[\widehat{\varphi}_{n}(x, y)\right]=F(x, y)$ it follows from (1) that

$$
\begin{gathered}
\mathrm{E}\left[\widehat{F}_{n}(x, y)\right]=\int_{\mathbb{R}^{2}} U\left(\frac{x-s}{h_{n}}, \frac{y-t}{h_{n}}\right) d F(s, t)= \\
=\int_{\mathbb{R}^{2}} u(w, v) F\left(x-w h_{n}, y-v h_{n}\right) d w d v .
\end{gathered}
$$

Using a Taylor expansion of order 2 of $F$ and taking account of (A2) and (A4), derivatives of the theorem follows.

Note that assumptions (A2) and (A4) are only required in order to establish a convergence rate. In fact, $\mathrm{E}\left[\widehat{F}_{n}(x, y)\right] \longrightarrow F(x, y)$ is a consequence of the Dominated Convergence Theorem.

In order to establish the almost sure convergence of $\widehat{F}_{n}$ we will apply a strong law of large numbers proved by Newman (1980). In course of proof we will need to control some covariances.

Lemma 3.2 Suppose the variables $X_{n}, n \in \mathbb{N}$, satisfy (A1), (A2), (A3) and (A4). Then, for each $j \in \mathbb{N}$ and $x, y \in[0,1]$,

$$
\begin{aligned}
\operatorname{Cov}\left[U\left(\frac{x-X_{1}}{h_{n}}, \frac{y-X_{k+1}}{h_{n}}\right),\right. & \left.U\left(\frac{x-X_{j}}{h_{n}}, \frac{y-X_{k+j}}{h_{n}}\right)\right]= \\
& =F_{j}(x, y, x, y)-F^{2}(x, y)+O\left(h_{n}^{2}\right) .
\end{aligned}
$$

Proof : To treat the covariances we are reduced to study the behaviour of

$$
\int_{\mathbb{R}^{4}} U\left(\frac{x-s}{h_{n}}, \frac{y-t}{h_{n}}\right) U\left(\frac{x-w}{h_{n}}, \frac{y-v}{h_{n}}\right) d F_{j}(s, t, w, v) .
$$

Using the same arguments as described in the proof of Theorem 3.1 and its result, the lemma follows.

We may now prove the almost sure convergence of the estimator (1).

Theorem 3.3 Suppose the variables $X_{n}, n \in \mathbb{N}$, satisfy (A1), (A2), (A3), (A4), (A6') and (A7). Then, for every $x, y \in[0,1], \widehat{F}_{n}(x, y) \longrightarrow F(x, y)$ almost surely.

Proof : The variables $U\left(\frac{x-X_{m}}{h_{n}}, \frac{y-X_{k+m}}{h_{n}}\right), m \in \mathbb{N}$, are associated, as $U$ is coordinatewise nondecreasing, and stationary. Then, according to Newman (1980), they verify a strong law of large numbers if

$$
\lim _{n \rightarrow \infty} \frac{1}{n-k} \sum_{j=1}^{n-k} \operatorname{Cov}\left[U\left(\frac{x-X_{1}}{h_{n}}, \frac{y-X_{k+1}}{h_{n}}\right), U\left(\frac{x-X_{j}}{h_{n}}, \frac{y-X_{k+j}}{h_{n}}\right)\right]=0 .
$$

Now, from Lemma 3.2 and using an inequality by $\mathrm{Yu}$ (1993), it follows

$$
\begin{aligned}
\operatorname{Cov}\left[U\left(\frac{x-X_{1}}{h_{n}}, \frac{y-X_{k+1}}{h_{n}}\right), U\left(\frac{x-X_{j}}{h_{n}}, \frac{y-X_{k+j}}{h_{n}}\right)\right] \leq \\
\leq 3^{1 / 3} 2^{5 / 3} \operatorname{Cov}^{1 / 3}\left(X_{1}, X_{j}\right)
\end{aligned}
$$


so that (2) follows from (A6') and association.

In fact, we may prove the uniform consistency of the estimator under the same set of conditions.

Theorem 3.4 Suppose the variables $X_{n}, n \in \mathbb{N}$, satisfy (A1), (A2), (A3), (A4), (A6') and (A7). Then

$$
\sup _{x, y \in[0,1]}\left|\widehat{F}_{n}(x, y)-F(x, y)\right| \longrightarrow 0 \quad \text { a.s. }
$$

Proof : The proof follows the usual steps: decompose $[0,1]^{2}$ using a fixed set of suitably chosen points and establish convenient inequalities. Let $M>$ 1 be fixed. Then

$$
\Delta_{M, n}=\max _{0 \leq i, j \leq M}\left|\widehat{F}_{n}\left(\frac{i}{M}, \frac{j}{M}\right)-F\left(\frac{i}{M}, \frac{j}{M}\right)\right| \longrightarrow 0 \quad \text { a.s.. }
$$

Now, using that $\widehat{F}_{n}$ is nondecreasing and that the $X_{n}, n \in \mathbb{N}$, are uniformly $[0,1]$ distributed, it follows easily that, if $x \in\left[\frac{i-1}{M}, \frac{i}{M}\right)$ and $y \in\left[\frac{j-1}{M}, \frac{j}{M}\right)$,

$$
\left|\widehat{F}_{n}(x, y)-F(x, y)\right| \leq \Delta_{M, n}+\frac{2}{M},
$$

from which the theorem follows, as $M$ is arbitrary.

\section{THE MEAN SQUARE ERROR}

In this section we look at the convergence rate of the mean square error, from which we derive the optimal bandwidth convergence rate of order $n^{-1}$, thus a different convergence rate than the one for the independent case. This confirms a different behaviour of the bandwidth when compared with the independent case, already noticed in Cai, Roussas (1998).

As usual we write

$$
\begin{aligned}
\operatorname{MSE}\left(\widehat{F}_{n}(x, y)\right)=\mathrm{E}[ & \left.\left(\widehat{F}_{n}(x, y)-F(x, y)\right)^{2}\right]= \\
& =\operatorname{Var}\left(\widehat{F}_{n}(x, y)\right)+\left(\mathrm{E}\left[\widehat{F}_{n}(x, y)\right]-F(x, y)\right)^{2} .
\end{aligned}
$$

The behaviour of $\mathrm{E}\left[\widehat{F}_{n}(x, y)\right]$ being known, we need to describe the asymptotics and convergence rate for

$$
\begin{aligned}
& \operatorname{Var}\left(\widehat{F}_{n}(x, y)\right)=\frac{1}{n-k} \operatorname{Var}\left[U\left(\frac{x-X_{1}}{h_{n}}, \frac{y-X_{k+1}}{h_{n}}\right)\right]+ \\
& \quad+\frac{2}{(n-k)^{2}} \sum_{j=2}^{n-k}(n-k-j+1) \operatorname{Cov}\left[U\left(\frac{x-X_{1}}{h_{n}}, \frac{y-X_{k+1}}{h_{n}}\right), U\left(\frac{x-X_{j}}{h_{n}}, \frac{y-X_{k+j}}{h_{n}}\right)\right] .
\end{aligned}
$$


The behaviour of all these terms has been described in Lemma 3.2. Just notice that, the variance term, which corresponds to Lemma 3.2 with $j=1$, gives as limit $F_{1}(x, y, x, y)-F^{2}(x, y)=F(x, y)-F^{2}(x, y)$.

We may now insert these convergence rates in (3), from which the next result is an immediate consequence.

Theorem 4.1 Suppose the variables $X_{n}, n \in \mathbb{N}$, satisfy (A1), (A2), (A3), (A4), (A5), (A6) and (A7). Then

$$
\begin{aligned}
& (n-k) \operatorname{MSE}\left(\widehat{F}_{n}(x, y)\right)=F(x, y)-F^{2}(x, y)+ \\
& \quad+2 \sum_{j=2}^{\infty}\left(F_{j}(x, y, x, y)-F^{2}(x, y)\right)+O\left(h_{n}+n h_{n}^{2}\right)+a_{n}
\end{aligned}
$$

where

$a_{n}=\frac{1}{n-k} \sum_{j=2}^{\infty}(j-1)\left(F_{j}(x, y, x, y)-F^{2}(x, y)\right)-2 \sum_{j=n-k-1}^{\infty}\left(F_{j}(x, y, x, y)-F^{2}(x, y)\right)$.

Note that $a_{n} \longrightarrow 0$ according to the assumptions made and that $a_{n}$ is independent of the bandwidth choice. It is now evident that an optimization of the convergence rate of the MSE is achieved by choosing $h_{n}=c n^{-1}$.

\section{FINITE DIMENSIONAL DISTRIBUTIONS}

To prove the asymptotic normality of the finite dimensional distributions of the estimator we will use a decomposition of the sums into several blocks that are associated. These are then replaced by independent ones with the same distributions. This approximation is controlled via Newman's inequality (see Newman (1984)). As the proof is somewhat long, we will divide it in some lemmas. Before proceeding define, for every $x, y, w, v \in[0,1]$,

$$
\begin{aligned}
& \alpha_{n}(x, y)=\sqrt{n-k}\left(\widehat{F}_{n}(x, y)-F(x, y)\right) \\
& \sigma^{2}(x, y, w, v)=F\left(\min (x, w), \min _{\infty}^{\infty}(y, v)\right)-F(x, y) F(w, v)+ \\
& +2 \sum_{j=2}^{\infty}\left(F_{j}(x, y, w, v)-F(x, y) F(w, v)\right) .
\end{aligned}
$$

Throughout this section we will suppose that the random variables $X_{n}, n \in$ $\mathbb{N}$, satisfy (A1), (A2), (A3), (A4), (A5), (A6) and (A7).

Lemma 5.1 For every $x, y, w, v \in[0,1]$,

$$
\operatorname{Cov}\left(\alpha_{n}(x, y), \alpha_{n}(w, v)\right) \rightarrow \sigma^{2}(x, y, w, v) .
$$


Proof : Use the stationarity to decompose $\operatorname{Cov}\left(\alpha_{n}(x, y), \alpha_{n}(w, v)\right)$ into a sum and apply to each term the methods used in the proofs of Lemma 3.2 and Theorem 4.1.

For the lemmas concerning directly the proof of the asymptotic normality we need some further notation. First denote $\widetilde{n}=n-k$ and, given an integer $r \leq \widetilde{n}$, let $m$ be the largest integer less or equal to $\widetilde{n} / r$. Let $s \in \mathbb{N}$, $c_{1}, \ldots, c_{s} \in \mathbb{R}, x, y, x_{1}, \ldots, x_{s}, y_{1}, \ldots, y_{s} \in[0,1]$ and define

$$
\begin{gathered}
T_{\widetilde{n}, i}(x, y)=U\left(\frac{x-X_{i}}{h_{n}}, \frac{y-X_{k+i}}{h_{n}}\right)-\mathrm{E}\left[U\left(\frac{x-X_{i}}{h_{n}}, \frac{y-X_{k+i}}{h_{n}}\right)\right] \\
Y_{j}^{r}(x, y)=\frac{1}{\sqrt{r}} \sum_{i=(j-1) r+1}^{j r} T_{\widetilde{n}, i}(x, y), \quad W_{j}^{r}=\sum_{q=1}^{s} c_{q} Y_{j}^{r}\left(x_{q}, y_{q}\right) . \\
Z_{\widetilde{n}}=\frac{1}{\widetilde{n}} \sum_{q=1}^{s} c_{q} \sum_{i=1}^{\widetilde{n}} T_{\widetilde{n}, i}\left(x_{q}, y_{q}\right), \quad Z_{m r}=\frac{1}{\sqrt{m}} \sum_{q=1}^{s} c_{q} \sum_{j=1}^{m} Y_{m r}\left(x_{q}, y_{q}\right) .
\end{gathered}
$$

Note that, as follows from Lemmas 3.2 and 5.1,

$$
\operatorname{Var}\left(Z_{\widetilde{n}}\right) \longrightarrow \sigma^{2}:=\sum_{q=1}^{s} c_{q}^{2} \sigma^{2}\left(x_{q}, y_{q}, x_{q}, y_{q}\right)+2 \sum_{q=1}^{s-1} \sum_{l=q+1}^{s} c_{q} c_{l} \sigma^{2}\left(x_{q}, y_{q}, x_{l}, y_{l}\right)
$$

and, for $r$ fixed,

$$
\operatorname{Var}\left(W_{j}^{r}\right)=\sigma_{r}^{2}:=\sum_{q=1}^{s} c_{q}^{2} \sigma_{r}^{2}\left(x_{q}, y_{q}, x_{q}, y_{q}\right)+2 \sum_{q=1}^{s-1} \sum_{l=q+1}^{s} c_{q} c_{l} \sigma_{r}^{2}\left(x_{q}, y_{q}, x_{l}, y_{l}\right)
$$

where

$$
\begin{aligned}
\sigma_{r}^{2}\left(x_{q}, y_{q}, x_{l}, y_{l}\right)=F( & \left.\min \left(x_{q}, x_{l}\right), \min \left(y_{q}, y_{l}\right)\right)-F\left(x_{q}, y_{q}\right) F\left(x_{l}, y_{l}\right) \\
& +\frac{2 r-2}{r} \sum_{j=2}^{r}\left(F_{j}\left(x_{q}, y_{q}, x_{l}, y_{l}\right)-F\left(x_{q}, y_{q}\right) F\left(x_{l}, y_{l}\right)\right) .
\end{aligned}
$$

We proceed now to the lemmas into which the proof of Theorem 5.5 below is divided. First, replace the sum up to $\widetilde{n}$ by the sum up to $m r$ to find a sum consisting only on the blocks $W_{j}^{r}$.

Lemma 5.2 For fixed $r,\left|\mathrm{E} e^{i t Z_{n}}-\mathrm{E} e^{i t Z_{m r}}\right| \longrightarrow 0$.

Proof : From Hölder's inequality it follows

$$
\begin{aligned}
\left|\mathrm{E} e^{i t Z_{\tilde{n}}}-\mathrm{E} e^{i t Z_{m r}}\right|^{2} & \leq 4 t^{2} \mathrm{E}\left[\left(Z_{\widetilde{n}}-Z_{m r}\right)^{2}\right] \leq \\
& \leq 4 t^{2}\left[2\left(\frac{1}{\sqrt{m r}}-\frac{1}{\sqrt{\widetilde{n}}}\right)^{2} \operatorname{Var}\left(Z_{\widetilde{n}}\right)+\frac{8(\widetilde{n}-m r)^{2}}{m r}\left(\sum_{q} c_{q}\right)^{2}\right]
\end{aligned}
$$


which converges to zero, according to the assumptions made.

Now, we control the approximation between our sum and what we would find if the blocks $W_{j}^{r}$ were independent.

Lemma 5.3 For $r$ fixed, there exists a constant $c>0$, such that

$$
\begin{aligned}
\left|\mathrm{E} e^{i t Z_{m r}}-\mathrm{E} e^{\frac{i t}{\sqrt{m}} W_{j}^{r}}\right| \leq & c t^{2} \sum_{q=1}^{s}\left|\sigma_{r}^{2}\left(x_{q}, y_{q}, x_{q}, y_{q}\right)-\sigma^{2}\left(x_{q}, y_{q}, x_{q}, y_{q}\right)\right| \\
& +2 t^{2} \sum_{q=1}^{s-1} \sum_{p=q+1}^{s}\left|\sigma_{r}^{2}\left(x_{q}, y_{q}, x_{p}, y_{p}\right)-\sigma^{2}\left(x_{q}, y_{q}, x_{p}, y_{p}\right)\right|
\end{aligned}
$$

Proof : This is a consequence of Newman's inequality and asymptotics of the variances of $Z_{\tilde{n}}$ and of $W_{j}^{r}$ mentioned above.

Lemma 5.4 For $r$ fixed, $\left|\prod_{j=1}^{m} \mathrm{E} e^{\frac{i t}{\sqrt{m}} W_{j}^{r}}-e^{\frac{t^{2} \sigma_{r}^{2}}{2}}\right| \longrightarrow 0$.

Proof : Just apply the Lindeberg condition to the variables $\frac{1}{\sqrt{m}} W_{j}^{r}, j=$ $1, \ldots, m$, and take account of the fact that each $W_{j}^{r}$ is a sum of $r$ bounded variables, so it is also bounded.

Finally the theorem that characterizes the asymptotic normality.

Theorem 5.5 The random vector $\left(\alpha_{n}\left(x_{1}, y_{1}\right), \ldots, \alpha_{n}\left(x_{s}, y_{s}\right)\right)$ converges in distribution to a gaussian centered random vector with covariance matrix

$$
\Gamma=\left[\begin{array}{cccc}
\sigma^{2}\left(x_{1}, y_{1}, x_{1}, y_{1}\right) & \sigma^{2}\left(x_{1}, y_{1}, x_{2}, y_{2}\right) & \cdots & \sigma^{2}\left(x_{1}, y_{1}, x_{q}, y_{q}\right) \\
\sigma^{2}\left(x_{2}, y_{2}, x_{1}, y_{1}\right) & \sigma^{2}\left(x_{2}, y_{2}, x_{2}, y_{2}\right) & \cdots & \sigma^{2}\left(x_{2}, y_{2}, x_{q}, y_{q}\right) \\
\cdots & \cdots & \cdots & \cdots \\
\sigma^{2}\left(x_{q}, y_{q}, x_{1}, y_{1}\right) & \sigma^{2}\left(x_{q}, y_{q}, x_{2}, y_{2}\right) & \cdots & \sigma^{2}\left(x_{q}, y_{q}, x_{q}, y_{q}\right)
\end{array}\right]
$$

Proof : From the Lemmas 5.2, 5.3 and 5.4, it follows that, for $r$ fixed,

$$
\begin{aligned}
\mid \mathrm{E} e^{i t Z_{\tilde{n}}}- & e^{\frac{t^{2} \sigma^{2}}{2}}\left|\leq c t^{2} \sum_{q=1}^{s}\right| \sigma_{r}^{2}\left(x_{q}, y_{q}, x_{q}, y_{q}\right)-\sigma^{2}\left(x_{q}, y_{q}, x_{q}, y_{q}\right) \mid \\
& +2 t^{2} \sum_{q=1}^{s-1} \sum_{p=q+1}^{s}\left|\sigma_{r}^{2}\left(x_{q}, y_{q}, x_{p}, y_{p}\right)-\sigma^{2}\left(x_{q}, y_{q}, x_{p}, y_{p}\right)\right|+\left|e^{\frac{t^{2} \sigma_{r}^{2}}{2}}-e^{\frac{t^{2} \sigma^{2}}{2}}\right| .
\end{aligned}
$$

Now letting $n \rightarrow \infty$, this converges to zero, so the theorem follows.

This work has been partially supported by CMAT and FCT under the program POCI 2010. 


\section{References}

Cai, Z., Roussas, G. (1998) Efficient Estimation of a Distribution Function under Quadrant Dependence. Scand. J. Statist., 25, 211-224.

Henriques, C., Oliveira, P.E. (1999) Covariance estimation for associated random variables. Preprint. Dep. Matemática Univ. Coimbra, 99-03.

Jin, Z., Shao, Y. (1999) On kernel estimation of a multivariate distribution function. Statist. Probab. Lett., 41, 163-168.

Newman, C.M. (1980) Normal fluctuations and the FKG inequalities. Comm. Math. Phys., 75, 119-128.

Newman, C.M. (1984) Asymptotic independence and limit theorems for positively and negatively dependent random variables. IMS Lecture Notes - Monograph Series, 5, 127-140.

Oliveira, P.E., Suquet, C. (1995) $L^{2}[0,1]$ weak convergence of the empirical process for dependent variables. Lecture Notes Statist., 103, 331-344.

Yu, H. (1993) A Glivenko-Cantelli lemma and weak convergence for empirical processes of associated sequences. Probab. Theory Related Fields, 95, 357370.

Roussas, G. (1995) Asymptotic normality of a smooth estimate of a random field distribution function under association. Statist. Probab. Lett., 24, 77-90. 